\title{
Introduction of a system to screen for chronic kidney disease and monitor for nephrotoxicity of antiretrovirals at an outpatient HIV clinic

\author{
S Kelly*1, C Bergin ${ }^{1}$, V Treacy ${ }^{1}$, L Sahm² and E de Barra ${ }^{1}$
}

Address: ${ }^{1}$ St James's Hospital/University College Cork, Dublin, Ireland and ${ }^{2}$ University College, Cork, Ireland

* Corresponding author

from Ninth International Congress on Drug Therapy in HIV Infection

Glasgow, UK. 9-13 November 2008

Published: 10 November 2008

Journal of the International AIDS Society 2008, I I (SuppI I):PI 32 doi:I0.II86/I758-2652-I I-SI-PI 32

This abstract is available from: http://www.jiasociety.org/content/I I/SI/PI32

(c) 2008 Kelly et al; licensee BioMed Central Ltd.

\section{Purpose of the study}

To introduce and audit the effectiveness of a system to screen for chronic kidney disease (CKD) and monitor for nephrotoxicity of antiretrovirals in HIV-positive patients.

\section{Methods}

Aging population, co-morbidities, antiretroviral therapies and HIV infection increase the risk of renal morbidity. The frequency of screening for CKD, and the frequency of monitoring for nephrotoxicity of antiretrovirals in HIVpositive patients attending outpatient clinics was audited. Of patients who required regular monitoring of GFR/urinalysis based on their antiretroviral therapy or risk factors for CKD, $0 \%$ of patients had a documented estimation of GFR ( $\mathrm{n}=31), 51.6 \%$ had urinalysis at an appropriate frequency $(\mathrm{n}=31), 11.76 \%$ had serum phosphate measurement at an appropriate frequency $(n=17)$. A series of interventions were implemented to highlight the recommended frequency of screening and monitoring, and allow quick identification of patients requiring urinalysis/ GFR estimation/serum phosphate measurement (tenofovir). A traffic light colour-coded system was used to group patients into three categories based on the frequency at which they required urinalysis/GFR estimation. The categories were based on recommendations from the IDSA and a review of current literature. Red, amber and green stickers were attached to each patient's chart indicating the need for 3-monthly/at next appointment screening, 6monthly screening or yearly screening, respectively. Educational presentations, posters, and an amended system to order bloods, which included an option for eGFR measurement, were provided to highlight and facilitate the recommended screening schedule.

\section{Summary of results}

Preliminary results indicate that the system has resulted in an improvement in screening for CKD and monitoring for nephrotoxicity of antiretrovirals.

\section{Conclusion}

Improved screening will allow identification of patients who require interventions to reduce cardiovascular risk and progression of CKD, timely referral and planning for renal replacement therapy, and early identification of nephrotoxicity due to antiretrovirals.

\section{References}

I. Gupta SK, et al.: Guidelines for the management of chronic kidney disease in HIV-infected patients: recommendations of the HIV Medicine Association of the Infectious Diseases Society of America. Clin Infect Dis 2005, 40(I I): 1559-85. 